\title{
AVALIAÇÃO DA FUNÇÃO PULMONAR APÓS COLECISTECTOMIAS LAPAROSCÓPICAS E CONVENCIONAIS
}

\author{
PULMONARY PERFORMANCE TEST AFTER CONVENTIONAL AND LAPAROSPIC \\ CHOLECISTECTOMY
}

\author{
Gilson Cassem Ramos'; ${ }^{1}$ Edísio Pereira ${ }^{2}$; \\ Salustiano Gabriel Neto, TCBC-GO ${ }^{3}$; Enio Chaves de Oliveira, TCBC-GO ${ }^{4}$
}

\begin{abstract}
RESUMO: Objetivo: Avaliar a função pulmonar pós-colecistectomias laparoscópicas e subcostais abertas. Métodos: Tratou-se de um ensaio randomizado, onde se avaliaram espirometrias pós-operatórias de dois grupos, cada qual com 15 pacientes. O grupo GL foi submetido a colecistectomia laparoscópica. O grupo GA foi submetido a colecistectomia por via subcostal, por meio de mini-laparotomia e abreviado tempo anestésico-cirúrgico. As variáveis dos dois grupos foram comparadas entre si por meio da ANOVA. Entre um mesmo grupo, antes e depois das operações, utlizou-se do teste $t$-Student emparelhado. Um valor de $\mathrm{p}<0,05$ foi considerado estatisticamente significativo. Resultados: Todas as pacientes, dos dois grupos, apresentaram distúrbios ventilatórios restritivos pós-operatórios, com normalização espirométrica mais rápida nas pacientes operadas por laparoscopia. Grupos GL X GA, no pós-operatório imediato: Capacidade vital forçada $(\mathrm{p}<0,001)$ e Volume Expiratório forçado em 1 segundo $(\mathrm{p}<0,001)$. Conclusões: O prejuízo pós-operatório da função pulmonar foi significativamente menor nas colecistectomias laparoscópicas do que nas abertas, mesmo por meio de minilaparotomia e abreviado tempo anestésico-cirúrgico (Rev. Col. Bras. Cir. 2007; 34(5): 326-330).
\end{abstract}

Descritores: Testes de função respiratória; Colecistectomia; Colecistectomia laparoscópica.

\section{INTRODUÇÃO}

Alguns procedimentos cirúrgicos interferem na mecânica pulmonar e tendem a desenvolver alterações ventilatórias restritivas, com redução do Volume Expiratório Forçado no $1^{\circ}$ segundo $\left(\mathrm{VEF}_{1}\right)$ e da Capacidade Vital Forçada $(\mathrm{CVF})$, podendo esta última variável atingir valor de, aproximadamente, 40 a $50 \%$ do valor pré-operatório e permanecer reduzida por, no mínimo, uma a duas semanas ${ }^{1}$.

Na maioria dos procedimentos cirúrgicos abdominais, esses desarranjos atingem seu pico no primeiro dia de pós-operatório ${ }^{2,3}$, momento em que o sistema respiratório se torna mais vulnerável a complicações pulmonares pós-operatórias ${ }^{4}$. Essas alterações ocorrem, especialmente, em operações no andar superior do abdome e é determinada principalmente pela disfunção diafragmática, desencadeada pelo estímulo cirúrgico ${ }^{5,6}$.

Dentre outros fatores que corroboram para a inibição diafragmática encontram-se a duração do ato anestésicocirúrgico e o tamanho da incisão cirúrgica. Dessa forma, procedimentos cirúrgicos abertos promovem as mais intensas alterações ventilatórias, que, contudo, são também observados em procedimentos laparoscópicos ${ }^{7}$. A técnica laparoscópica é a de primeira escolha para as colecistectomias, entretanto em algumas situações clínicas, ou por dificuldades técnicas, a via laparoscópica é substituída pela via aberta.

Assim, nesses casos, a mini-laparotomia, com tempo anestésico-cirúrgico abreviado, poderia amenizar os distúrbios ventilatórios restritivos pós-colecistectomias por promover disfunções diafragmáticas menos intensas. $\mathrm{O}$ presente estudo comparou dois grupos de pacientes se submeteram à colecistectomia: um por via laparoscópica e o outro por via aberta com incisão subcostal. Os objetivos foram: a) detectar, em ambos os grupos, distúrbios ventilatórios restritivos pósoperatórios, o período em que eles se tornam mais intensos e determinar sua gravidade e b) averiguar o período para a obtenção de espirometrias pós-operatórias normais.

\section{MÉTODO}

Este estudo foi realizado em pacientes que procuraram o Serviço de Cirurgia do Aparelho Digestivo do Hospital Ortopédico de Goiânia, após ter sido aprovado pelo Comitê de Ética em Pesquisa do Hospital de Urgências de Goiânia e de ter se obtido por escrito o Termo de Consentimento Livre e Esclarecido dos enfermos envolvidos na pesquisa.

Foram escolhidos aleatoriamente 30 pacientes de uma população de não vulneráveis, do Sistema Único de Saúde

1. Título Superior em Anestesiologia; Especialista em Clínica Médica; Doutor em Medicina pela UnB-DF; Responsável Pelo Serviço de Anestesia do Hospital Samaritano de Goiânia-GO.

2. Professor Doutor do Programa de Pós-Graduação da Faculdade de Ciências da Saúde da UnB - DF.

3. Cirurgião do Aparelho Digestivo; Professor Assistente de Técnica Operatória da Faculdade de Medicina da UFG - GO; Mestre em Cirurgia.

4. Professor Doutor da Disciplina de Coloproctologia da Faculdade de Medicina da UFG - GO.

Recebido em 06/03/2007

Aceito para publicação em 07/05/2007

Conflito de interesses: nenhum

Fonte de financiamento: nenhuma

Trabalho realizado e vinculado ao Programa de Pós-graduação em Ciências da Saúde da UnB-DF. 
(SUS), do gênero feminino, com idade entre 21 e 65 anos, com Índice de Massa Corpórea (IMC) menor ou igual a 35, estado físico ASA I ou II, com exames pré-operatórios normais e que se submeteriam à colecistectomia.

Excluiram-se enfermas em uso de fármacos com efeito broncodilatador, tabagistas, pacientes cuja estatura não pode ser determinada com precisão (cifoescoliose, amputação de membros inferiores etc.), gestantes, portadoras de doenças respiratórias, abdome agudo, história médica pregressa de doença diverticular do cólon, enfermas com úlcera gastroduodenal, pacientes com antecedentes clínicos de hemorragia digestiva, portadoras de doença neuro-muscular, pacientes psiquiátricas, com contra-indicação de receberem bloqueio anestésico peridural e atencedentes clínicos de alergia à dipirona, diclofenaco de sódio ou aos anestésicos selecionados para uso.

Tratou-se de um estudo experimental do tipo ensaio clínico randomizado. As pacientes foram distribuídas em dois Grupos, de 15 componentes, alocadas por sorteio. Todas foram submetidas à colecistectomia sob bloqueio anestésico peridural e anestesia geral. As do grupo-controle GL foram operadas por laparoscopia, as do Grupo-estudo GA, pela via aberta subcostal por mini-laparotomia (incisão de $6 \mathrm{~cm}$ ) e abreviado tempo antestésico-cirúrgico, de forma a não ultrapassar 50 minutos, inclusive com extubação oro-traqueal.

As anestesias foram padronizadas. Na sala de operação, cada enferma recebeu previamente 5 a 10mg de diazepam, depois de punção venosa periférica. A hidratação foi mantida com solução eletrolítica balanceada $\left(8 \mathrm{ml} \cdot \mathrm{kg}^{\mathrm{h}} \mathrm{h}^{-1}\right)$. A monitorização constou de ECG contínuo na derivação DII, oximetria de pulso, capnografia, frequiência cardíaca e pressão arterial com esfigmomanômetro. A paciente era posicionada em decúbito lateral esquerdo. Após infiltração local com 50 mg de lidocaína $1 \%$ no espaço intervertebral lombar L1-2, realizava-se punção peridural com agulha de Tuohy $16 \mathrm{G}$ e bisel voltado cefalicamente. Administrou-se nas pacientes, de todos os grupos, 93,75mg (25 ml de solução anestésica local) de bupivacaína $0,375 \%$ com adrenalina (1:200.000). O nível ou altura do bloqueio sensitivo promovido pela anestesia peridural foi avaliado pelo critério de Hollmen ${ }^{8}$. As anestesias foram induzidas com tionembutal $\left(5 \mathrm{mg} \cdot \mathrm{kg}^{-1}\right)$, fentanil $\left(3 \mu \mathrm{g} . \mathrm{kg}^{-}\right.$ $\left.{ }^{1}\right)$ e atracúrio $\left(0,5 \mathrm{mg} \cdot \mathrm{kg}^{-1}\right)$ e mantidas com isofluorano $(0,5 \mathrm{a}$ $1 \%$ ) e $\mathrm{N}_{2} \mathrm{O}$ numa mistura de $50 \%$ com $\mathrm{O}_{2}$. A intubação orotraqueal foi com cânula número $7,5 \mathrm{~mm}$, com insuflação do balonete com $5 \mathrm{ml}$ de ar. Foram administrados dipirona, $2 \mathrm{~g}$, endovenosamente e diclofenaco de sódio, $75 \mathrm{mg}$, intramuscular respectivamente de seis em seis horas e de 12 em 12 horas, iniciando na alta da sala de recuperação pós-anestésica, até 48 horas após o procedimento cirúrgico.

As pacientes foram operadas sempre pelo mesmo cirurgião, com a mesma técnica cirúrgica, tanto para as laparoscopias quanto para as abertas.

As pacientes submeteram-se à espirometrias seriadas. A primeira espirometria era realizada no pré-operatório. A segunda, no dia seguinte ao procedimento, entre as primeiras 24 horas de pós-operatório. A partir deste momento, a cada dois dias realizaram-se novos exames, até a obtenção de um teste normal. As espirometrias foram realizadas sempre pela mesma profissional, Técnica em Função Pulmonar, e utilizando o mesmo aparelho: espirômetro portátil Spiro Pro ${ }^{\circledR}$ versão 2.0. Trata-se de um aparelho de fabricação Norte Americana, validado pela American Thoracic Societ, capaz de medir parâmetros pulmonares de fluxo $\left(\right.$ l.seg $\left.^{-1}\right)$ e volume (1). Esse equipamento além de gerar as curvas de fluxo-volume e de volume-tempo, discrimina variáveis espirométricas, com os valores preditos baseados na equação de regressão de Knudson ${ }^{9}$.

A preparação para cada sessão de espirometria foi baseada em normativas da Sociedade Brasileira de Pneumologia e Tisiologia ${ }^{10}$ e incluía calibrar o espirômetro com seringa apropriada de 31, e ajustá-lo, de acordo com a temperatura ambiente $\left(25 \mathrm{a} 40^{\circ} \mathrm{C}\right)$ e pressão atmosférica $(680 \mathrm{mmHg})$. Cada paciente submetia-se a três testes válidos e reprodutíveis. $\mathrm{O}$ aparelho Spiro Pro ${ }^{\circledR}$ versão 2.0 utiliza o maior valor obtido da equação $\mathrm{CVF}+\mathrm{VEF}_{1}$ para selecionar o melhor teste.

Os laudos das espirometrias foram fornecidos sempre pelo mesmo médico pneumologista, que os interpretava sem conhecer a história clínica da paciente.

A prova broncodilatadora foi incluída na rotina das espirometrias uma vez que a Técnica em Função Pulmonar foi orientada a seguir sua prática habitual. Contudo, os dados obtidos pós-broncodilatação não foram analisados.

As variáveis $\mathrm{CVF}$ e $\mathrm{VEF}_{1}$ foram analisadas, pré e pósoperatoriamente, até o momento de suas normalizações (80\% do valor teórico pré-calculado para $\mathrm{CVF}_{\mathrm{e}} \mathrm{VEF}_{1}$ e em torno de $70 \%$ para a relação $\left.\mathrm{VEF}_{1} / \mathrm{CVF}\right)$.

Para a análise de hipótese de igualdade de médias entre os dois grupos, utilizou-se a técnica de análise da variância-ANOVA ${ }^{11}$. A hipótese de igualdade de médias entre um mesmo grupo, antes e após as colecistectomias, foi verificada por meio do teste $t$-Student emparelhado. Um valor de $p<0,05$ foi considerado estatisticamente significativo.

\section{RESULTADOS}

A Tabela 1 refere-se às características individuais das pacientes estudadas e variáveis operatórias. Observaram-se diferenças estatisticamente significativas para a variável altura $(\mathrm{p}=0,021)$, duração da Intubação oro-traqueal $(\mathrm{p}<0,001)$ e tempo cirúrgico $(\mathrm{p}<0,001)$.

A Tabela 2 refere-se aos valores da variável CVF e do $\mathrm{VEF}_{1}$, do pré-operatório até o $3^{\circ}$ dia de pós-operatório. Em relação aos valores pré-operatórios, não existiram diferenças estatisticamente significativas. Para a CVF existiram diferenças estatisticamente significativas, quando se comparou o pós-operatório imediato $(\mathrm{p}=0,000)$ e o $3^{\circ}$ dia de pós-operatório $(\mathrm{p}=0,037)$. Em relação ao $\mathrm{VEF}_{1}$, existiram diferenças altamente significativas no pós-operatório imediato $(\mathrm{p}=0,000) \mathrm{e}$ no $3^{\circ}$ dia de pós-operatório $(\mathrm{p}=0,000)$. Figura1 representa a ilustração gráfica da evolução da CVF, ao longo desse período.

A Tabela 3 refere-se à comparação das variáveis espirométricas de um mesmo grupo, antes e depois das colecistectomias. Foram observadas diferenças estatisticamente significativas em todas as comparações, exceto para CVF (p $=0,689)$ do grupo GL, quando se comparou a média pré-opera- 
Tabela 1 - Características das pacientes estudadas e variáveis operatórias (média \pm desvio padrão).

\begin{tabular}{|c|c|c|c|c|}
\hline \multirow[t]{2}{*}{ Características } & \multicolumn{2}{|c|}{ Grupos } & \multicolumn{2}{|c|}{ Estatística } \\
\hline & GL & GA & $\mathbf{F}$ & $\mathbf{p}$ \\
\hline Idade(anos) & $44,13 \pm 11,17$ & $44,67 \pm 10,31$ & 0,02 & 0,0892 \\
\hline Altura $(\mathrm{cm})$ & $153,20 \pm 2,23$ & $156,27 \pm 4,32$ & 5,98 & 0,021 \\
\hline Peso(kg) & $65,33 \pm 9,36$ & $67,48 \pm 11,99$ & 0,30 & 0,588 \\
\hline $\mathrm{IMC}^{*}$ & $27,66 \pm 4,10$ & $27,45 \pm 4,56$ & 0,02 & 0,895 \\
\hline Incisão $(\mathrm{cm})$ & $\longrightarrow$ & $6,2 \pm 0,41$ & & \\
\hline Duração da IOT**(min) & $40,80 \pm 6,68$ & $28,80 \pm 3,63$ & 37,37 & $<0,001$ \\
\hline Tempo cirúrgico(min) & $24,60 \pm 6,52$ & $16,33 \pm 4,73$ & 15,81 & $<0,001$ \\
\hline
\end{tabular}

* IMC = peso $(\mathrm{kg}) /$ altura $\left(\mathrm{m}^{2}\right) ;$ min = minutos.

**IOT = Intubação oro-traqueal.

Tabela 2 - Valor médio da CVF e VEF, litros. Pré-operatório até o $3^{\circ}$ dia de pós-operatório (média \pm desvio padrão).

\begin{tabular}{|c|c|c|c|c|c|c|}
\hline \multirow[t]{2}{*}{ Grupos } & \multicolumn{2}{|c|}{ CVF } & \multicolumn{2}{|c|}{$\mathrm{VEF}_{1}$} & \multicolumn{2}{|c|}{ Estatística (valor de p) } \\
\hline & GL & GA & GL & GA & $\begin{array}{c}\text { Pré-operatório } \\
\text { X PO imediato } \\
(\mathrm{CVF})\end{array}$ & $\begin{array}{c}\text { Pré-operatório } \\
\text { X 30 PO }^{\circ} \\
\left(\mathrm{VEF}_{1}\right) \\
\end{array}$ \\
\hline Pré-operatório & $3,30 \pm 0,32$ & $3,22 \pm 0,48$ & $2,73 \pm 0,33$ & $2,62 \pm 0,44$ & 0,000 & 0,037 \\
\hline PO imediato & $3,03 \pm 0,42$ & $2,06 \pm 0,68$ & $2,50 \pm 0,38$ & $1,63 \pm 0,55$ & & \\
\hline $3^{\circ} \mathrm{PO}$ & $3,33 \pm 0,28$ & $2,86 \pm 0,78$ & $2,89 \pm 0,26$ & $2,15 \pm 0,61$ & 0,000 & 0,000 \\
\hline
\end{tabular}

PO = pós-operatório.

tória com a do $3^{\circ}$ dia de pós-operatório. Para o $\mathrm{VEF}_{1}$, nesta comparação, a média foi maior no $3^{\circ}$ dia, em relação ao valor pré-operatório. A Figura 2 ilustra a redução da CVF no préoperatório imediato, nos dois grupos.

A normalização das espirometrias, comparadas aos valores preditos, ocorreu no pós-operatório imediato, no terceiro, quinto e sétimo dias de pós-operatório, respectivamente, em 33,33, 46,70, 13,3 e 6,7\% das pacientes do Grupo GA e no pós-operatório imediato em $100 \%$ das do Grupo GL. No Grupo GL e GA os bloqueios peridurais estabeleceram-se entre os dermátomos T2 e T4.

\section{DISCUSSÃO}

No presente estudo observou-se a presença de distúrbios ventilatórios restritivos leves (Tabela 3), mais intensos no pós-operatório imediato, com redução da CVF e do $\mathrm{VEF}_{1}$, nos dois grupos de pacientes, quando essas duas variáveis foram comparadas pré e pós-operatoriamente (Tabelas 2 e Figura 2).

Esse achado vem ao encontro de vários experimentos que relatam a ocorrência de distúrbios ventilatórios restritivos após operações no andar superior de abdome, especialmente, no primeiro dia de pós-operatório ${ }^{12}$. O grupo de pacientes GL apresentou menores alterações espirométricas pósoperatórias, observação que também se identifica com outras publicações científicas ${ }^{13-15}$, porém nesta pesquisa, a diminuição mais acentuada da CVF e do $\mathrm{VEF}_{1}$, nesse grupo, foi, respectivamente, de $8,2 \%$ e $8,4 \%$ em relação aos valores pré-

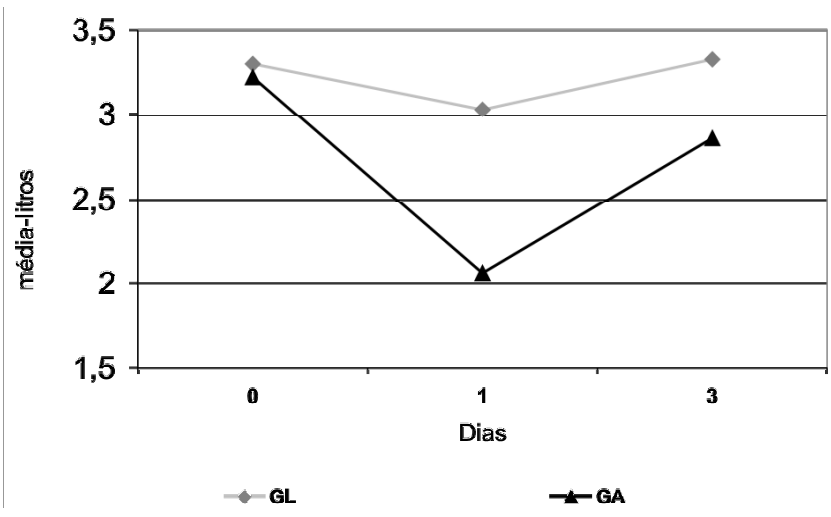

Figura 1 - CVF : do período pré-operatório até o terceiro dia de pósoperatório.

operatórios (Tabela 2). Isso equivale a dizer que os valores espirométricos pós-operatórios nas laparoscopias são de exames normais, comparados aos valores preditos. Não foram encontrados relatos de observações científicas equivalentes aos verificados nas pacientes do grupo GL. O que se verifica, habitualmente, são alterações mais pronunciadas, mesmo nas laparoscopias, com reduções entre $20 \%$ e $30 \%{ }^{16,17}$ em ambas as variáveis ou até mesmo quedas mais significativas de mais de $40 \%{ }^{18}$.

Os distúrbios ventilatórios observados no pós-operatório imediato das pacientes do grupo GA foram mais intensos que os apurados no grupo GL, com as variáveis espirométricas $\mathrm{CVF}_{\text {e }} \mathrm{VEF}_{1}$ significativamente reduzidas $(\mathrm{p}=$ 
Tabela 3 - Valor médio da CVF e do $V E F_{1}$ : do pré-operatório ao $3^{\circ}$ dia de pós-operatório (média \pm DP).

\begin{tabular}{|c|c|c|c|c|c|c|}
\hline \multirow[t]{2}{*}{ Grupos } & \multicolumn{2}{|c|}{ CVF } & \multicolumn{2}{|c|}{$\mathbf{V E F}_{1}$} & \multicolumn{2}{|c|}{ Estatística (valor de p) } \\
\hline & GL & GA & GL & GA & $\begin{array}{c}\text { Pré-operatório } \\
\text { X PO imediato } \\
\text { (CVF) }\end{array}$ & $\begin{array}{c}\text { Pré-operatório } \\
\mathbf{X} 3^{\circ} \mathbf{P O} \\
\left(\mathrm{VEF}_{1}\right) \\
\end{array}$ \\
\hline Pré-operatório & $3,30 \pm 0,32$ & $3,22 \pm 0,48$ & $2,73 \pm 0,33$ & $2,62 \pm 0,44$ & $0,000 / 0,000$ & $0,020 / 0,022$ \\
\hline PO imediato & $3,03 \pm 0,42$ & $2,06 \pm 0,68$ & $2,50 \pm 0,38$ & $1,63 \pm 0,55$ & & \\
\hline $3^{\circ} \mathrm{PO}$ & $3,33 \pm 0,28$ & $2,86 \pm 0,78$ & $2,89 \pm 0,26$ & $2,15 \pm 0,61$ & $0,023 / 0,008$ & $0,689 / 0,049$ \\
\hline
\end{tabular}

$P O=$ pós-operatório.

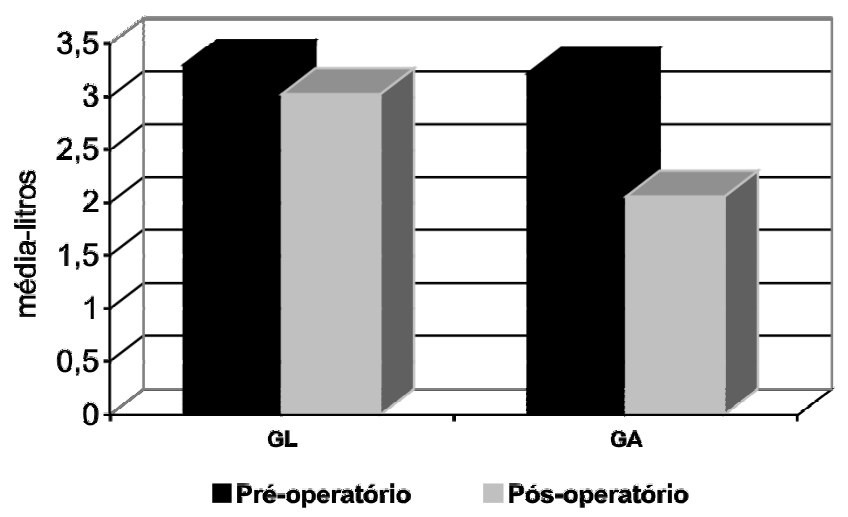

Figura 2 - CVF: pré-operatório e pós-operatório imediato.

0,001) em relação às pacientes tratadas por via laparoscópica (Tabelas 2 e 3), e semelhantes aos aferidos em outras pesqui$\operatorname{sas}^{1}$. A redução média dos parâmetros $\mathrm{CVF}$ e $\mathrm{VEF}_{1}$, no pósoperatório imediato, foi, respectivamente, de 36,02\% e 38,16\%, em relação aos valores pré-operatórios (Tabelas 2 e 3).

A duração dos procedimentos cirúrgicos abertos no grupo GA apresentou-se significativamente menor $(\mathrm{p}<0,001)$ em relação ao grupo GL (Tabela 1). Além disso, a incisão subcostal nos procedimentos abertos foi de mini-laparotomia ${ }^{19}$, em torno de $6 \mathrm{~cm}$ (Tabela 1 ).

Um dos propósitos da presente pesquisa não foi o de verificar o tempo de recuperação das espirometrias pósoperatórias aos níveis pré-operatórios e, sim, a normalização desses exames de acordo com os valores preditos. Outros estudos notificaram que a recuperação da função pulmonar pós-operações de abdome superior varia de sete a 14 dias $^{1,2}$, período de maior vulnerabilidade pulmonar ${ }^{4}$. No entanto, é de se especular se pacientes que alcançam provas espirométricas pós-operatórias normais em relação aos valores preditos, porém inferiores às pré-operatórias, estariam mais sujeitos às complicações pulmonares.

Todas as pacientes do grupo GL apresentaram, nos pós-operatórios imediatos, espirometrias consideradas normais, porém inferiores aos valores obtidos no período préoperatório. Assim, nesse grupo as variáveis espirométricas $\mathrm{CVF}_{\mathrm{V}} \mathrm{VEF}_{1}$ diminuíram dos valores pré em relação aos valores atingidos no pós-operatório imediato, e na medida seguinte (terceiro dia de pós-operatório), os valores obtidos já se equivaleram aos do pré-operatório (Figura 1).
Algumas pesquisas apontaram para uma recuperação da função pulmonar pós-colecistectomia laparoscópica entre oito a dez dias ${ }^{20,21}$, o que não encontra amparo no presente estudo. Provavelmente, essa diferença poderia ser justificada pelo tempo cirúrgico abreviado, com uma injúria tecidual e disfunção diafragmática menores nas pacientes do grupo GL. As pacientes do grupo GA completaram as normalizações espirométricas no sétimo dia de pós-operatório.

Algumas variáveis metodológicas podem tornar difícil a comparação desta investigação científica com outras, no que diz respeito à normalização espirométrica. Por exemplo, o espirômetro utilizado neste estudo orientou-se pela equação de regressão de Knudson ${ }^{9}$, que foi publicada em 1983. Já, pesquisa relevante ${ }^{2}$, anterior a essa data, foi orientada por outro método. Os valores preditos conduziram à normalização das espirometrias, enquanto outros pautaram pela busca de valores referenciais pré-operatórios ${ }^{2}$. Entretanto, ao avaliar o grupo GA, não se pode afirmar que houve tendência de antecipação da normalização da função pulmonar. Tal afirmação necessitaria de ser confirmada em uma população-estudo maior. Por outro lado pode-se afirmar que, nas pacientes do grupo GL observaram-se menores reduções pós-operatórias das variáveis $\mathrm{CVF}^{\mathrm{e}} \mathrm{VEF}_{1}$, com tendência à normalização espirométrica precoce.

ACVFé o principal parâmetro respiratório na detecção dos distúrbios ventilatórios restritivos. Neste experimento, o valor médio dessa variável, nos dois grupos, caracterizou distúrbios pós-operatórios leves (Tabela 3), contudo algumas pacientes do grupo GA apresentaram distúrbios mais importantes. Por exemplo, uma paciente do grupo GA evoluiu no primeiro pós-operatório com redução da CVF de, aproximadamente, $92 \%$. Essas constatações mostram o risco potencial das complicações pós-operatórias a que os pacientes estão sujeitos, dentre os quais insuficiência respiratória aguda e morte ${ }^{22,23}$.

A técnica laparoscópica mostrou ser superior aos procedimentos abertos, mesmo os realizado por minilaparotomia e abreviado tempo anestésico-cirúrgico. Dessa forma, a incisão cirúrgica (subcostal) próxima ao diafragma é um fator causal mais importante do que o próprio tamanho da incisão e o tempo operatório juntos, na gênese da disfunção deste músculo (não aferida nesta pesquisa) e, conseqüentemente, dos distúrbios ventilatórios pós-operatórios. Então, mesmo nas indicações de colecistectomias por via aberta ocorre uma limitação dessa técnica cirúrgica, no sentido de proteção pulmonar, o que, certamente, pode aumentar a morbidade pós-operatória. 


\begin{abstract}
Background: To evaluate pulmonary function after laparoscopic and subcostal cholecystectomies . Methods: This was a randomized study, in which postoperative spirometries in two groups of fifteen patients each were evaluated. Group GL underwent laparoscopic chlecystectomies. Group GA underwent open subcostal cholecystectomies by means of mini-laparatomy, in abbreviated anesthetic-surgical time. The two groups'variables were compared using ANOVA. Within the same group, before and after the operations, the paired Student- $t$ test was used. A value of $p<0.05$ was considered statistically significant. Results: All patients from both groups presented restrictive postoperative ventilatory disturbances, with a faster spirometric normalization for those patients operated through laparoscopy. Groups GLvs. GA, in immediate post-operative: Forced Vital Capacity $(p<0.001)$ and Forced Expiratory Volume in one second $(p<0.001)$. Conclusion: Postoperative harm to pulmonary function was significantly less in laparoscopic than in open cholecystectomies, even with mini-laparotomies and abbreviated anesthetic-surgical time.
\end{abstract}

Key words: Respiratory function tests; Cholecystectomy; Laparoscopic cholecystectomy.

\section{REFERÊNCIAS}

1. Craig DB. Postoperative recovery of pulmonary function. Anesth Analg. 1981;60(1):46-52.

2. Ali J, Weisel RD, Layug AB, Kripke BJ, Hechtman HB. Consequences of postoperative alterations in respiratory mechanics. Am J Surg. 1974;128(3):376-82.

3. Dureuil B, Cantineau JP, Desmonts JM. Effects of upper or lower abdominal surgery on diaphragmatic function. Br J Anaesth. 1987;59(10):1230-5.

4. Fairshter RD, Williams JH Jr. Pulmonary physiology in the postoperative period. Crit Care Clin. 1987;3(2):287-306.

5. Simonneau G, Vivien A, Sartene R, Kunstlinger F, Samii K, Noviant Y, Duroux P. Diaphragm dysfunction induced by upper abdominal surgery. Role of postoperative pain. Am Rev Respir Dis. 1983;128(5):899-903

6. Ford GT, Whitelaw WA, Rosenal TW, Cruse PJ, Guenter CA. Diaphragm function after upper abdominal surgery in humans. Am Rev Respir Dis. 1983;127(4):431-6.

7. Erice F, Fox GS, Salib YM, Romano E, Meakins JL, Magder SA. Diaphragmatic function before and after laparoscopic cholecystectomy. Anesthesiology. 1993;79(5):966-75; discussion 27A-28A.

8. Büttner J, Klose R. [Alkalinization of mepivacaine for axillary plexus anesthesia using a catheter.] Reg Anaesth. 1991;14( 1):1724.

9. Knudson RJ, Lebowitz MD, Holberg CJ, Burrows B. Changes in the normal maximal expiratory flow-volume curve with growth and aging. Am Rev Respir Dis. 1983;127(6):725-34.

10. Sociedade Brasileira de Pneumologia e Tisiologia. I Consenso brasileiro sobre espirometria. J Pneumol. 1996;22(3):121-8.

11. Beiguelman B. Curso prático de bioestatística. Rev Bras Gen. 1996;165-94.

12. Aboussouan LS, Stoller JK. Perioperative pulmonary care. In: Cherniak NS, Altose MD, Homma I. Rehabilitation of patient with respiratory disease. New York: Mc Graw - Hill; 1999. p. 561-75.

13. Johnson D, Litwin D, Osachoff J, McIntosh D, Bersheid B, Church D, Yip R, Gallagher C. Postoperative respiratory function after laparoscopic cholecystectomy. Surg Laparosc Endosc. 1992;2(3):221-6.

14. Mahul P, Burgard G, Costes F, Guillot B, Massardier N, el Khouri Z, Cuilleret J, Geyssant A, Auboyer C. [Postoperative respiratory function and cholecystectomy by laparoscopic approach.] Ann Fr Anesth Reanim. 1993;12(3):273-7.

15. Hasukic S, Mesic D, Dizdarevic E, Keser D, Hadziselimovic S, Bazardzanovic M. Pulmonary function after laparoscopic and open cholecystectomy. Surg Endosc. 2002;16(1):163-5. Epub 2001 Oct 19.

16. Frazee RC, Roberts JW, Okeson GC, Symmonds RE, Snyder SK, Hendricks JC, Smith RW. Open versus laparoscopic cholecystectomy. A comparison of postoperative pulmonary function. Ann Surg. 1991;213(6):651-3; discussion 653-4.

17. Ravimohan SM, Kaman L, Jindal R, Singh R, Jindal SK. Postoperative pulmonary function in laparoscopic versus open cholecystectomy: prospective, comparative study. Indian J Gastroenterol. 2005;24(1):6-8.

18. Barnett RB, Clement GS, Drizin GS, Josselson AS, Prince DS. Pulmonary changes after laparoscopic cholecystectomy. Surg Laparosc Endosc. 1992;2(2):125-7.

19. Mcmahon AJ, Russell IT, Ramsay G, Sunderland G, Baxter JN, Anderson JR, Galloway D, O’Dwyer PJ. Laparoscopic and minilaparotomy cholecystectomy: a randomized trial comparing postoperative pain and pulmonary function. Surgery. 1994;115(5):533-9.

20. De La Peña M, Togores B, Bosch M, Maimo A, Abad S, Garrido P, Soro JA, Agustí AG. [Recovery of lung function after laparoscopic cholecystectomy: the role of postoperative pain.] Arch Bronconeumol. 2002;38(2):72-6.

21. Bablekos GD, Roussou T, Rasmussen T, Vassiliou MP, Behrakis PK. Postoperative changes on pulmonary function after laparoscopic and open cholecystectomy. Hepatogastroenterology. 2003;50(53):1193-200.

22. Kroenke K, Lawrence VA, Theroux JF, Tuley MR. Operative risk in patients with severe obstructive pulmonary disease. Arch Intern Med. 1992;152(5):967-71.

23. Wong DH, Weber EC, Schell MJ, Wong AB, Anderson CT, Barker SJ. Factors associated with postoperative pulmonary complications in patients with severe chronic obstructive pulmonary disease. Anesth Analg. 1995;80(2):276-84.

Como citar este artigo:

Ramos GC, Pereira E, Gabriel Neto S, Oliveira EC. Avaliação da função pulmonar após colecistectomias laparoscópicas e convencionais. Rev Col Bras Cir. [periódico na Internet] 2007 Set-Out; 34(5). Disponível em URL: www.scielo.br/rcbc

Endereço para correspondência:

Gilson Cassem Ramos. Rua 8, N. 74, Ap. 402

Setor Oeste

74117-100 - Goiânia-GO

E-mail: gilson.ramos@terra.com.br 